ISSN 1648-4142 TRANSPORT

http:/www.vtu.lt/english/editions

TRANSPORT - 2003, Vol XVIII, No I, 9-12

\title{
SOME METHODS OF IMPROVING FREIGHT TRANSPORTATION IN SHORT HAULS
}

\author{
Danutè Bagdonienè \\ Dept of Mathematical Statistics, Vilnius Gediminas Technical University, Sauletekio al. 11, LT-2040 Lithuania \\ E-mail: genmil@ti.vtu.lt
}

Received 200206 27; accepted 20021221

\begin{abstract}
The results obtained in the investigation aimed to improve the transportation of commercial freight for short hauls in the city are presented. The performed tasks included the statistical analysis of freight flows; the forecast of the freight flows; a synthesis of a topological structure of the urban system of freight transportation and the description of the optimal structure of the truck stock. The conclusions have also been drawn and some recommendations provided.
\end{abstract}

Keywords: freight transportation; short hauls.

\section{Introduction}

It seems that the improvement of transportation may be achieved by merely replacing the available transport facilities with the new ones. However, even most advanced vehicles may be inefficiently used. Therefore, transportation technology as a whole should be improved. This requires a thorough analysis of the problems associated with freight flows, their updating and forecasting, optimization of the road network and traffic, freight distribution, etc. The description of the problems referring to improving freight transportation over long distances and carriage of freight reaching 20-40 tons may be found in [1-4]. However, the data on the analysis of transportation of freight about $50-1500 \mathrm{~kg}$ in short hauls in a small area (i.e. with the restrictions imposed) is scarce. Therefore, we will try to suggest some solutions to these problems in the present work. First, the following tasks were performed:

1) the statistical analysis of the flows of freight was made;

2) forecasting of freight flows was carried out;

3) the structure of the stock of trucks of optimal tonnage was outlined;

4) freight consignments and the ways of transportation were determined.

\section{Determining the Stock Structure of the Trucks of Optimal Tonnage}

The structure of truck stock based on tonnage should satisfy the requirements raised for transporting various lots of goods. Let us suppose that truck tonnage is expressed by a string $q_{1}, q_{2}, \ldots, q_{j}, \ldots, q_{m}$. In addition, the distribution of lots of goods according to their volume is known. The probability of occurrence of goods requiring the transportation by a truck of $q_{j}(j=1,2, \ldots, m-1)$ tonnage is $[5,6]$ :

$$
p_{j}=\left\{\begin{array}{l}
\left(q_{\gamma}\right)_{j} \\
\int q_{0} f(x) d x, \quad j=1 ; \\
\left(q_{\gamma}\right)_{j}(x) d x, \quad j>1, \\
\left(q_{\gamma}\right)_{j-1} f(x)
\end{array}\right.
$$

here, $f(x)$-distribution density of lots of goods according to size (volume).

The probability of occurrence when a lot of goods requiring the transportation by a $q_{m}$ tonnage truck making $i$ hauls $(i=1,2, \ldots)$ has to be transported is:

$$
p_{m, i}=\left\{\begin{array}{l}
\left(q_{\gamma}\right)_{m}(x) d x, \quad i=1 \\
\left\{\begin{array}{l}
f(x) \\
\left(q_{\gamma}\right)_{m-1} \\
i\left(q_{\gamma}\right)_{m}
\end{array}\right. \\
(i-1) f(x) d x, \quad i>1 .
\end{array}\right.
$$

The required number of trucks of the type $j$ $(j=1,2, \ldots, m-1)$ is as follows:

$$
A_{e j}=\frac{\bar{N}_{v . r .} p_{j}}{T_{n j}}\left(\frac{l_{g . e j}}{v_{t j} \beta_{j}}+t_{n p j}\right),
$$

here $\bar{N}_{v . r}$. the average number of the requirements for goods transportation in 24 hours. 
The number of $q_{m}$ tonnage trucks required is:

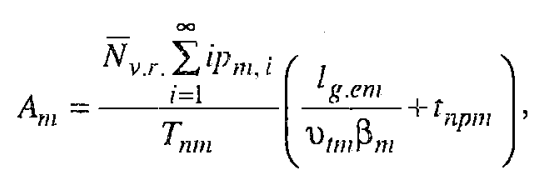

here, $T_{n m}$ - the work of $q_{m}$ tonnage truck expressed in hours.

The total number of trucks may be expressed in this way:

$$
\begin{aligned}
& A_{e}=\sum_{j=1}^{m} A_{e j}=\bar{N}_{v \cdot r}\left[\sum_{j=1}^{m-1} \frac{p_{j}}{T_{n j}}\left(\frac{l_{g . e j}}{v_{t j} \beta_{j}}+t_{n p j}\right)+\right. \\
& \left.\sum_{i=1}^{\sum_{n m}^{\infty} i p_{m, i}}\left(\begin{array}{c}
l_{g . e m} \\
T_{t m} \beta_{m}
\end{array}+t_{n p m}\right)\right] .
\end{aligned}
$$

By dividing the left and right - hand sides of the equations (3) and (5) we will obtain:

$$
\frac{A_{e j}}{A_{e}}=\frac{p_{j}}{T_{n j} B}\left(\begin{array}{c}
l_{g \cdot e j} \\
v_{t j} \beta
\end{array} t_{n j j}\right), \ldots j=1,2, \ldots, m-1
$$

Similariy, the equations (4) and (5) yield:

$$
\frac{A_{m}}{A_{e}}=\frac{\sum_{i=1}^{\infty} i p_{m, i}}{T_{n j} B}\left(\begin{array}{l}
l_{g \cdot e j} \\
v_{t j} \beta_{j}
\end{array}+t_{n p j}\right)
$$

while (6) and (7) yield:

$$
\begin{aligned}
& B=\frac{A_{e}}{N_{v . r}}=\sum_{j=1}^{m-1} P_{j j}\left(\frac{l_{g . e j}}{T_{n j}}+\iota_{n p j}\right)_{t} \beta_{j} \\
& \frac{\sum_{i=1}^{\infty} i p_{m, i}}{T_{n m}}\left(\frac{l_{g . e m}}{v_{m m} \beta_{m j}}+t_{n p m !}\right) .
\end{aligned}
$$

If $T_{n j}=T_{n m}=T_{i n}$, we have to calculate:

$$
\begin{aligned}
& T_{n} B=\sum_{j=1}^{m-1} p_{j}\left(\frac{l_{g . e j}}{v_{t j} \beta_{j}}+t_{n p j}\right)+ \\
& \left(\frac{l_{g . e m}}{v_{m m} \beta_{m}}+t_{n p m} \sum_{j=l}^{\infty} i p_{m, i} .\right.
\end{aligned}
$$

Thus, in order to determine the probability of the requirements for the transportation by trucks of various tonnage it is sufficient to identify the type of distribution of lots of goods according to their size and to know the average number of truck hauls in 24 hours.

Density function of exponential distribution of the volumes of goods lots may be expressed in this way:

$$
f(x)=\frac{1}{\bar{g}} e^{-\frac{x}{\bar{g}}}
$$

here $\bar{g}$ - average volume of a lot of goods

$$
\begin{aligned}
& p_{1}=\frac{1}{\bar{g}} \int_{0}^{\left(q_{\gamma}\right)_{1}} e^{-\frac{x}{\bar{g}}} d x=1-e^{-\frac{\left(q_{\gamma}\right)_{1}}{\bar{g}}}, \\
& p_{j}=\frac{1}{\bar{g}}\left(q_{q_{\gamma}}\right)_{j-1}^{\left(q_{\gamma}\right)_{j}} e^{-\frac{x}{\bar{g}}} d x=e^{-\frac{\left(q_{\gamma}\right)_{j-1}}{\bar{g}}}-e^{-\frac{\left(q_{\gamma}\right)_{j}}{\bar{g}}},
\end{aligned}
$$

$p_{m, i}=\frac{1}{\bar{g}}(i-1) \int_{\left(q_{\gamma}\right)_{m}}^{i\left(q_{\gamma}\right)_{m}} e^{-\frac{x}{\bar{g}}} d x=e^{-\frac{(i-1)\left(q_{\gamma}\right)_{m}}{\bar{g}}}-e^{-\frac{i\left(q_{\gamma}\right)_{m}}{\bar{g}}}$

If the volumes of lots of goods are distributed according to normal distribution, the probability of occurrence of an arbitrary value $q$ in the interval $\left[\left(g_{\gamma}\right)_{j-1},\left(q_{\gamma}\right)_{j}\right]$ may be determined in this way:

$$
\begin{aligned}
& p_{j}=P\left\{\left(q_{\gamma}\right)_{j-1}<g<\left(q_{\gamma}\right)_{j}\right\}=\Phi *\left[\frac{\left(q_{\gamma}\right)_{j}-\bar{g}}{\sigma_{g}}\right]- \\
& \Phi^{*}\left[\frac{\left(q_{\gamma}\right)_{j-1}-\bar{g}}{\sigma_{g}}\right],
\end{aligned}
$$

here, $\sigma_{g}$ is a standard deviation of an arbitrary value, while

$$
p_{m, i}=\left\{\begin{array}{l}
\Phi *\left[\frac{\left(q_{\gamma}\right)_{m}-\bar{g}}{\sigma_{g}}\right]-\Phi *\left[\frac{\left(q_{\gamma}\right)_{m-1}-\bar{g}}{\sigma_{g}}\right], \quad i=1 ; \\
\Phi *\left[\frac{i\left(q_{\gamma}\right)_{m}-\bar{g}}{\sigma_{g}}\right]-\Phi *\left[\frac{(i-1)\left(q_{\gamma}\right)_{m}-\bar{g}}{\sigma_{g}}\right], \quad i>1
\end{array}\right.
$$

In some cases the operators and senders relate the volume of the transported lots of goods to the tonnage of the truck. Then an average volume of the transported lot of goods will be:

$$
\bar{g}=\sum_{j=1}^{m-1} p_{j}\left(q_{\gamma}\right)_{j}+\left(q_{\gamma}\right)_{m} \sum_{i=1}^{\infty} i p_{m, i}
$$

here, $\left(q_{\gamma}\right)_{j},\left(q_{\gamma}\right)_{m}-$ the highest tonnage of the truck 
based on the capacity of the truck body and the type of freight.

An average volume of the lot of goods transported in a single haul is as follows:

$$
\bar{g}_{e}=\sum_{j=1}^{m-1} p_{j}\left(q_{\gamma}\right)_{j}+\left(q_{\gamma}\right)_{m} \sum_{i=1}^{\infty} p_{m, i}
$$

An average truck tonnage calculated for a single haul is:

$$
\dot{q}_{e}=\sum_{j=1}^{m-1} p_{j} q_{j}+q_{m} \sum_{i=1}^{\infty} p_{m, i}
$$

An average value of the static coefficient of using the tonnage of the truck stock may be expressed in this way:

$$
\gamma_{s t}=\frac{g_{e}}{q_{e}}=\frac{\sum_{j=1}^{m-1} p_{j}\left(q_{\gamma}\right)_{j}+\left(q_{\gamma}\right)_{m} \sum_{i=1}^{\infty} p_{m, i}}{\sum_{j=1}^{m-1} p_{j} q_{j}+q_{m} \sum_{i=1}^{\infty} p_{m, i}} .
$$

A number of hauls made by all trucks of the stock during the period investigated is:

$$
n_{e}=\frac{P}{\bar{q}_{e} \gamma_{s t}},
$$

here, $P$ - the total volume of transportation, ton.

The number of hauls made by $j$ type trucks is:

$$
n_{e j}=p_{j} n_{e}, \quad j=1,2, \ldots, m-1,
$$

while for the trucks of the highest tonnage it is:

$$
n_{e m}=n_{e} \sum_{i=1}^{\infty} p_{m, i}=n_{e}-\sum_{j=1}^{m-1} n_{e j}
$$

The volume of transportation by $q_{j}$ tonnage trucks is as follows:

$$
P_{j}=n_{e j}\left(q_{\gamma}\right)_{j}, j=1,2, \ldots, m .
$$

The number of the required $q_{j}$ tonnage trucks is:

$$
\bar{A}_{j}=\frac{P_{j}}{D \alpha_{j} P_{\text {par. } j}}, j=1,2, \ldots, m,
$$

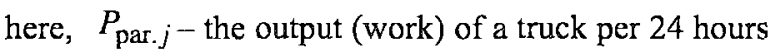

$$
P_{\text {par. } . j}=\frac{v_{t j} \beta_{j} q_{j} \gamma_{s t j} T_{n j}}{l_{g . e j}+v_{t j} \beta_{j} t_{n p j}} .
$$

Example. The volumes of the goods transported from the warehouses of manufacturers to a chain of shops have exponential distribution with density function (Fig):

$$
f(x)=0,0675 e^{-0,0675 x} .
$$

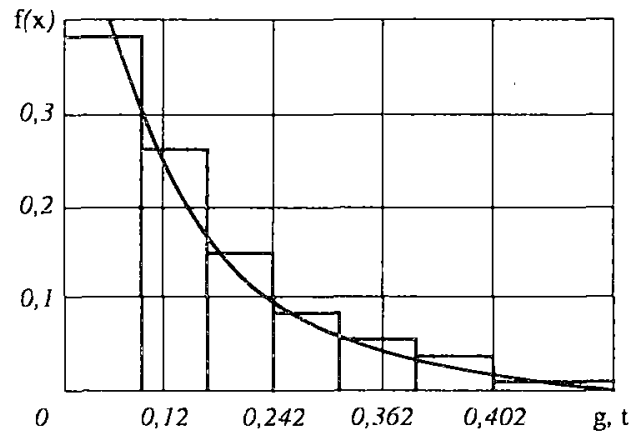

Distribution of lots of goods based on volume

An average volume of a lot of goods transported is $\bar{g}=1 / 0,0675=14,8$ i.e. $0,148 \mathrm{t}$ on. To transport $P=300$ ton of goods per year, $A_{1}$ trucks will be needed of the tonnage $q_{1}=350 \mathrm{~kg}, t_{n p l}=1,47, \alpha_{1}=0,75, A_{2}$ of the tonnage $q_{2}=700 \mathrm{~kg}, \alpha_{2}=0,79$, and $A_{3}$ of the tonnage $q_{3}=1500 \mathrm{~kg}, t_{n p 3}=1,58$ and $\alpha_{3}=0,74$. Other parameters are similar for all trucks: $l_{\text {r.ej }}=10 \mathrm{~km}$, $v_{t j}=22 \mathrm{~km} / \mathrm{h}, B_{j}=0,5, \cdots T_{n j}=12 \mathrm{~h}(j=1,2,3)$

The probability of occurrence of the requirements to transport lots of goods by trucks $A_{1}$, and $A_{2}$ is respectively as follows:

$$
\begin{aligned}
& p_{1}=1-e^{-\frac{\left(q_{\gamma}\right)_{1}}{\bar{g}}}=1-e^{-\frac{7,5}{14,8}}=0,4, \\
& p_{2}=e^{-\frac{\left(q_{\gamma}\right)_{1}}{\bar{g}}}-e^{-\frac{\left(q_{\gamma}\right)_{2}}{\bar{g}}}=e^{-\frac{7,5}{14,8}}-e^{-\frac{12,5}{14,8}}=0,17 .
\end{aligned}
$$

The probabilities of orders for transporting lots of goods by $A_{3}$ trucks in hauls $i=1,2, \ldots$, are equal to:

$$
\begin{aligned}
& p_{3,1}=e^{-\frac{\left(q_{\gamma}\right)_{2}}{\bar{g}}}-e^{-\frac{\left(q_{\gamma}\right)_{3}}{\bar{g}}}=e^{-12,5} \frac{14,8}{14}-e^{-\frac{14,0}{14,8}}=0,05, \\
& p_{3,2}=e^{-\frac{\left(q_{\gamma}\right)_{3}}{\bar{g}}}-e^{-\frac{2\left(q_{\gamma}\right)_{3}}{\bar{g}}}=e^{-\frac{14,0}{14,8}}-e^{-\frac{2 \cdot 14,0}{14,8}}=0,24, \\
& p_{3,3}=e^{-\frac{2\left(q_{\gamma}\right)_{3}}{\bar{g}}}-e^{-\frac{3\left(q_{\gamma}\right)_{3}}{\bar{g}}}=0,13, \\
& p_{3,4}=e^{-\frac{3\left(q_{\gamma}\right)_{3}}{\bar{g}}}-e^{-\frac{4\left(q_{\gamma}\right)_{3}}{\bar{g}}}=0,01, \\
& p_{3,5} \approx p_{3,6} \approx \ldots \approx 0 .
\end{aligned}
$$

In this case, $T_{H 1}=T_{H 2}=T_{H 3}$, therefore, we can find $T_{H} B$ from the formula (9).

A relative proportion of trucks based on their tonnage may be determined from the formulas (6) and (7):

$$
\frac{A_{E_{1}}}{A_{E}}=0,239 ; \frac{A_{E_{2}}}{A_{E}}=0,106 ; \frac{A_{E_{3}}}{A_{E}}=0,655 .
$$


A number of hauls performed by the trucks of the stock during the planned period may be obtained from the formula (19), $n_{e}=26600$, while the same parameter according to truck tonnage $n_{e 1}=10600 ; n_{e 2}=3110$; $n_{e 3}=12890$. An average number of trucks in a list according to their tonnage is as follows:

$A_{1}=7,71 ; A_{2}=2,4 ; \quad A_{3}=10,8 ;$ i.e. $A_{1} \approx 8 ;$ $A_{2} \approx 2 ; A_{3} \approx 11$.

\section{Conclusions}

1. To determine the optimal structure of the stock of trucks as well as organizing their efficient operation, lots of goods and the volume of transportation should be analyzed in terns of time. Since the requirements of transportation and the volume of lots of goods are arbitrary values, their analysis in time should be most efficiently made by means of mathematical statistics methods.

2. Methods of predicting the flows of freight have been chosen and updated on the basis of the performed statistical analysis of the freight flows. It has been shown that the best method of their prediction is based on mathematical expectation, statistical prediction relying on a single point being less accurate.

3. An algorithm derived for determining an optimal structure of truck stock based on tonnage allows the operator to select the required average number of trucks based on their tonnage.

4. The techniques for determining an optimal volume of the lots of goods transported and choosing the most suitable way of transportation based on such optimization criterion as total expenses on freight storage and transportation have been offered.

\section{References}

1. Guelat, J.; Florian, M.; Crainic, T.-G. A Multimode Multiproduct Network Assignment Model for Strategic Planning of Freight Flows. Transportation Science, Vol 24, No 1, February, 1990, p. 25-39.

2. Zhan, F.-B.; Noon, Ch.-E. Shortest Path Algorithms: An Evaluation using Real Road Networks. Transportation Science, Vol 32, No 1, February, 1998, p. 65-73.

3. Nuzzolo, A.; Russo, F.; Crisali, U. A Doubly Dynamic Schedule - based Assignment Model for Transit Network. Transportation Science, Vol 35, No 3, August, 2001, p. 268285.

4. Bodin, L.; Mingozzi, A.; Baldacci, R.; Ball, M. The Roll on - Rolloff Vehicle Routing Problem. Transportation Science, Vol 34, No 3, August, 2000, p. 256-271.

5. Baublys, A. Some methods of improving the transportation of commercial goods in short hauls in the city. In: Proceedings of international conference "Research and industry of the region of Aukštaitija". Kaunas: Technologija, 2002, p. $407-412$.

6. Baublys, A. Assessment of statistical probability of the technological transportation process. Transport, Vol XVII, No 4,2002, p. $127-136$. 